\title{
Pressure-Stimulated Supercrystals Formation in Nanoparticle Suspensions
}

Martin A. Schroer ${ }^{1, *}$, Felix Lehmkühler ${ }^{2,3, * *}$, Johannes Möller ${ }^{4}$, Holger Lange ${ }^{3,5}$, Gerhard Grübel $^{2,3}$ and Florian Schulz ${ }^{3,5, * * *}$

\section{AUTHOR ADDRESS}

${ }^{1}$ European Molecular Biology Laboratory (EMBL), Hamburg Outstation c/o DESY, 22607

Hamburg, Germany. ${ }^{2}$ Deutsches Elektronen-Synchrotron (DESY), 22607 Hamburg, Germany.

${ }^{3}$ The Hamburg Centre for Ultrafast Imaging (CUI), 22761 Hamburg, Germany. ${ }^{4}$ European XRay Free-Electron Laser Facility (XFEL), 22869 Schenefeld, Germany. ${ }^{5}$ University of Hamburg, Institute of Physical Chemistry, 20146 Hamburg, Germany.

\section{AUTHOR INFORMATION}

\section{Corresponding Author}

*e-mail: martin.schroer@embl-hamburg.de; **e-mail: felix.lehmkuehler@desy.de; ***e-mail: schulzf@chemie.uni-hamburg.de. 


\section{ABSTRACT}

Nanoparticles can self-organize into "supercrystals" with many potential applications. Different paths can lead to nanoparticle self-organization into such periodic arrangements. An essential step is the transition from an amorphous state to the crystalline one. In this manuscript we investigate how pressure can induce a phase-transition of a nanoparticle model system in water from the disordered liquid state to highly-ordered supercrystals. We observe a reversible pressure-induced supercrystals formation in concentrated solutions of gold nanoparticles by means of small angle X-ray scattering. The supercrystal formation occurs only at high salt concentrations in the aqueous solution. The pressure dependence of the structural parameters of the resulting crystal lattices is determined. The observed transition can be reasoned with the combined effect of salt and pressure on the solubility of the organic PEG-shell that passivates the nanoparticles.

\section{TOC GRAPHICS}

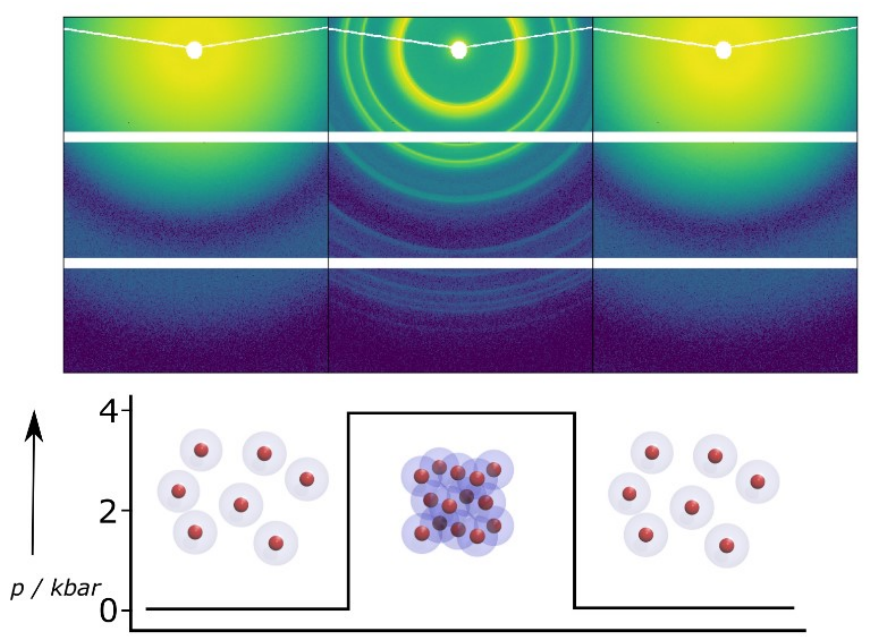


KEYWORDS Crystallization; high-pressure; Nanoparticle Suspensions; Phase transition; Small angle X-ray scattering;

The formation of nanoparticle (NP) supercrystals has been studied intensively throughout the last decades. ${ }^{1-5}$ The fact that NP supercrystals can exhibit tunable and collective properties that are different from the sum of its constituents is the main origin of interest in the crystallization processes. $^{6-11}$ The transition of NP in solution into crystalline structures has already been realized in various ways. ${ }^{3,12-15}$ Self-assembly and crystallization of NPs is generally a complex process, ${ }^{16-21}$ usually based on the controlled evaporation or precipitation of the NP-building blocks by slow addition of a non-solvent. ${ }^{3,22}$ These processes have to be slow in order to achieve homogenous crystallization conditions for obtaining high-quality supercrystals. Applying pressure as a homogenous method to induce various structural transformations and phase transitions in pre-ordered nanoparticle assemblies has only recently been done. ${ }^{23-27} \mathrm{Wu}$ et al. observed the pressure-induced annealing of gold nanoparticle (AuNP) assemblies into a crystalline phase with significantly less defects. ${ }^{26}$ Podsiadlo et al. studied the stability and elasticity of layered PbS-NP-supercrystals at high pressures. ${ }^{27}$ In another recent study, using preordered hetero-assemblies of NPs, pressure allowed defined structural transformations by interparticle fusion. ${ }^{25}$ All these studies, however, used already pre-ordered NP structures.

Based on our recent work and the established effect of salt on the solubility of AuNPs coated with poly(ethylene glycol)- (PEG-) based ligands, we present that well-defined crystallization of NPs by pressure changes cannot only be observed in NP assemblies, but already from aqueous NP solutions. This crystallization is fast, largely reversible and does not need the presence of pre-ordered crystals. We studied the crystallization in situ by small angle X-ray scattering (SAXS), varying the involved parameters: AuNP concentration, added salt type and 
concentration, and AuNP-stabilizing PEG-ligand length. The obtained pressure - salt concentration $(p-c)$ phase diagram reveals that the crystallization is clearly a result of the combined effect of salt and pressure on the PEG-coatings.

As a model system we employed AuNPs $\left(R_{\text {core }}=\sim 6 \mathrm{~nm}\right)$ coated with a PEG- based ligand that provides a high colloidal stability even at high particle concentrations. Two different ligand lengths $(5 \mathrm{kDa}, 2 \mathrm{kDa})$ were used to prepare AuNP@PEG conjugates with final particle concentrations of $4 \mu \mathrm{M}$ and $3 \mu \mathrm{M}$, corresponding to volume fractions of $6-10 \%$ (AuNP@PEG5k) and 1-3 \% (AuNP@PEG2k), respectively. Their syntheses were described previously. ${ }^{28-30}$ Upon evaporation of the solvent, AuNP@PEG can form extended solids with locally ordered domains. ${ }^{31}$ The addition of salt decreases the solubility of PEG in water and at high salt concentrations, aggregation of the free polymer can occur depending on the type of salt. ${ }^{32-34}$ Zámbó et al. demonstrated that the lower critical solution temperature of AuNP@PEG can be lowered effectively by the addition of $\mathrm{K}_{2} \mathrm{SO}_{4}{ }^{35}$ In addition, it was shown that AuNP@PEG in water can form fcc-superlattices at high concentrations of potassium carbonate, $\mathrm{K}_{2} \mathrm{CO}_{3}{ }^{36,37}$ Besides salt and temperature, pressure can also decrease the solubility of PEG. ${ }^{38,39}$ For AuNP@PEG we observed a pressure-induced deswelling of the PEG-ligand layer affecting interparticle interactions. ${ }^{40}$ Based on these observations and to explore a potential phase transition in solution, we prepared solutions of AuNP@PEG with different concentrations of $\mathrm{NaCl}, \mathrm{KCl}, \mathrm{RbCl}$ and $\mathrm{CsCl}$ and studied their response to pressure by SAXS.

Figure 1 displays 2D SAXS patterns recorded for a concentrated suspension of AuNPs coated with $5 \mathrm{kDa}$ PEG in $2 \mathrm{M} \mathrm{CsCl}$ solution at 1 bar, at 4000 bar, and after pressure release. At 1 bar, the isotropic scattering pattern shows a pronounced forward scattering, indicative of an 
amorphous NP suspension in the liquid state. For larger scattering angles, annuli are present that stem from the form factor of the AuNPs. When increasing the pressure to 4000 bar, DebyeScherrer rings appear in the SAXS pattern, demonstrating the pressure-induced formation of ordered supercrystals. After releasing the pressure, the SAXS pattern reverts to its original state.
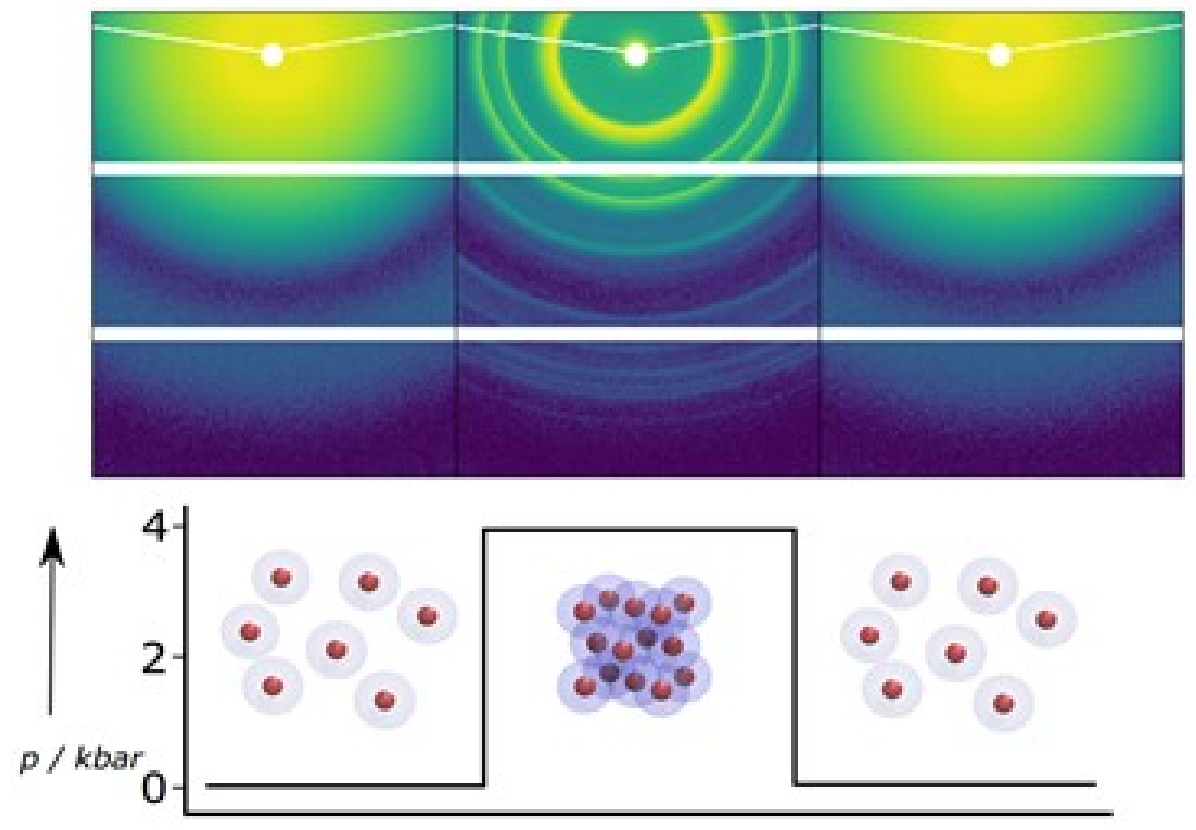

Figure 1. 2D SAXS patterns of PEG-coated AuNPs with $2 \mathrm{M} \mathrm{CsCl}$ added at different pressures. Left: 1 bar; Middle: 4000 bar; Right: After pressure release at 1 bar. The scheme at the bottom illustrates the structural assembly of the coated AuNPs at different pressures: At 1 bar, the particle ensemble is in an amorphous, liquid state. Upon reaching the crystallization pressure, face-centred cubic crystallites are formed by the AuNPs. After pressure release, the AuNPs return to the liquid state. 
This pressure-induced supercrystal formation takes place within a pressure-range of less than $\Delta p$ $=100 \mathrm{bar}$ as can be seen from the azimuthally integrated SAXS curves (Fig. 2a). For pressures from 1 bar up to 3000 bar, the SAXS curves do not show any indication of diffraction peaks, confirming the absence of crystalline order within the suspensions.

The pronounced oscillation at larger $q$ values stem from the form factor, $P(q)$, of the gold core with an average radius of $R_{\text {core }}=(6.25 \pm 0.05) \mathrm{nm}$, represented by solid lines. Only at small $q$, slight deviations to $P(q)$ are present, indicating the presence of a structure factor for liquids. As shown earlier for the AuNP@PEG system, the form factor represents basically the gold core, whereas the interparticle interactions are dominated by the PEG-layer. ${ }^{40,41}$ 


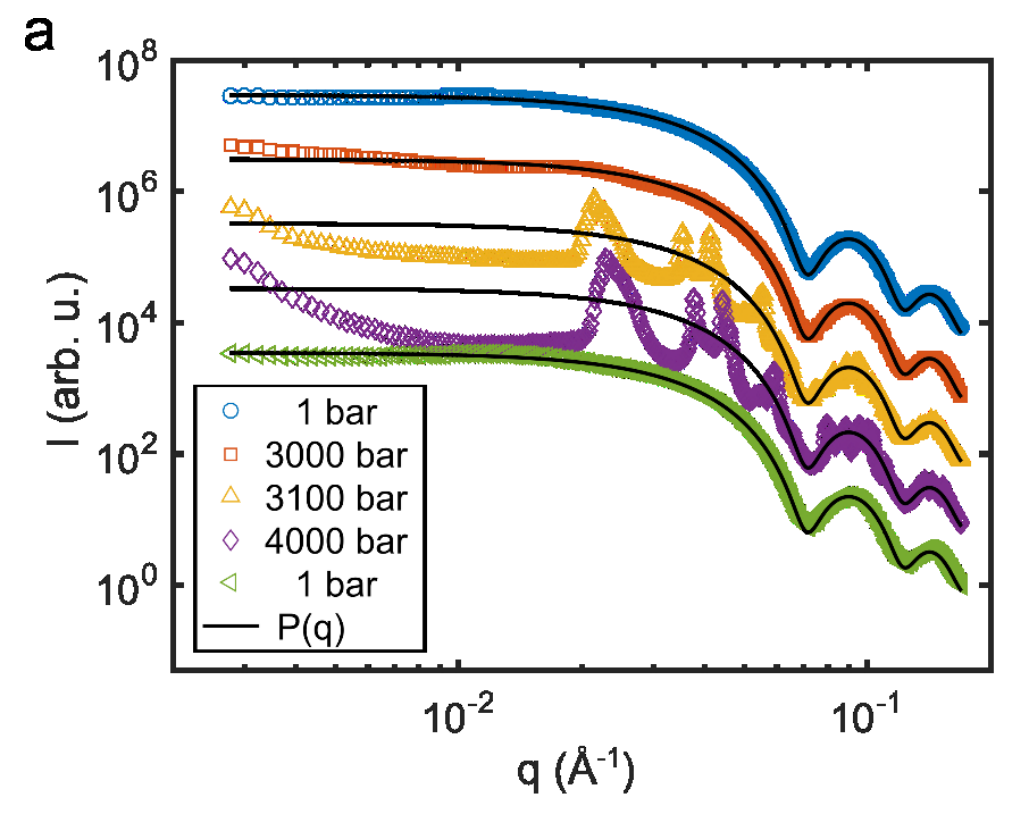

b

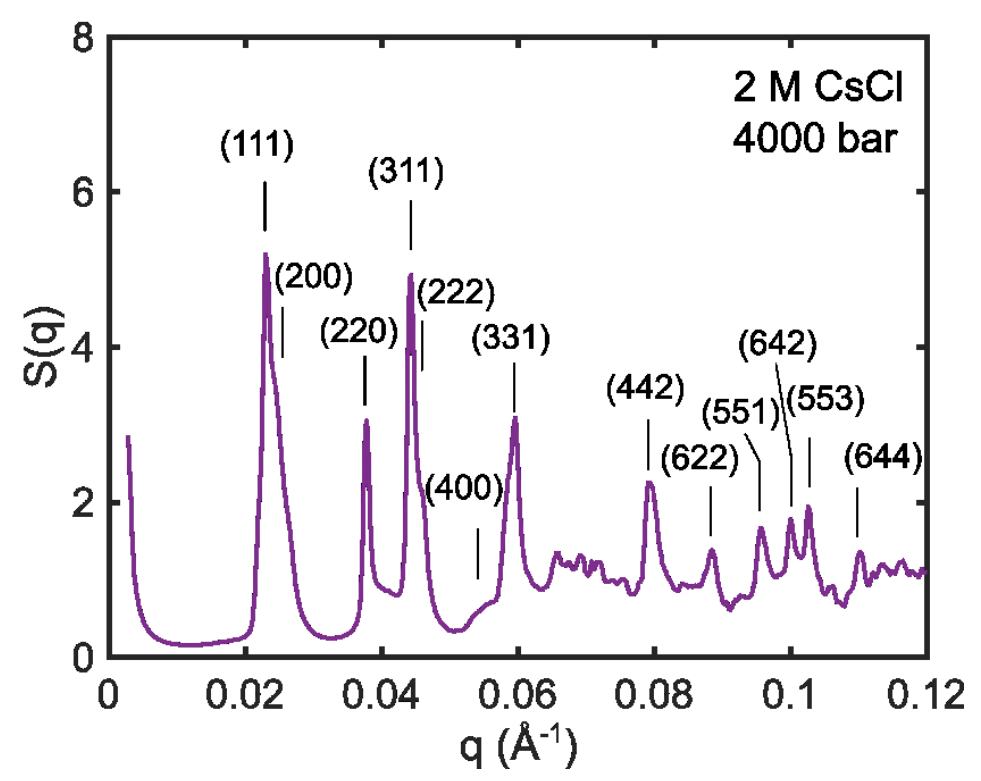

Figure 2. a) SAXS curves of a concentrated solution of AuNP@PEG with $2 \mathrm{M} \mathrm{CsCl}$ at different pressures. Starting from a pressure of 3100 bar (yellow triangles), Bragg reflections are present. At decompression the reflections disappear (green triangles). Solid lines indicate the form factor fit. b) Extracted structure factor for $2 \mathrm{M} \mathrm{CsCl}$ added to the suspension at 4000 bar. The reflections indicate a fcc structure. 
After increasing the pressure to 3100 bar, Bragg reflections appear. The reflections' positions shift to larger wave vectors $q$ with increasing pressure. The supercrystal structure factor $S(q)$ reveals a large set of Bragg reflections (>14 in the presented $q$-range) (Fig. 2b). The underlying crystal structure can be identified as a face-centered cubic lattice (fcc) which is formed by the periodic arrangement of the AuNPs. The large number of higher-order reflections indicates a high degree of crystallinity of the supercrystal, which is present already at the crystallization pressure of 3100 bar and does not significantly change with increasing pressure.

When reaching the crystallization pressure, supercrystals form within the whole suspension. The isotropic, continuous Debye-Scherrer rings indicate that these supercrystals are fully randomly orientated. After pressure release back to 1 bar, the reflections disappear, demonstrating a reversible transition.

The pressure-response of AuNP@PEG with different chloride salts $(\mathrm{NaCl}, \mathrm{KCl}, \mathrm{RbCl}, \mathrm{CsCl})$ at different concentrations shows a similar behavior (Fig. S1-S3). Except for $\mathrm{NaCl}$, pressureinduced crystallization sets in for salt concentrations from $1 \mathrm{M}$ onwards within the accessible pressure range from 1-4000 bar (see SI, experimental section). An increase in salt concentration leads to a continuous decrease of the crystallization pressure, whereas the lattice structure and degree of crystallinity is independent of the salt type and concentration. The crystallization is a sharp and fast phase transition ( $\Delta p<100$ bar, $\Delta t<30 \mathrm{~s}$ ) for all salt types studied. Based on these observations, a pressure - salt phase diagram was determined (Fig. 3). 


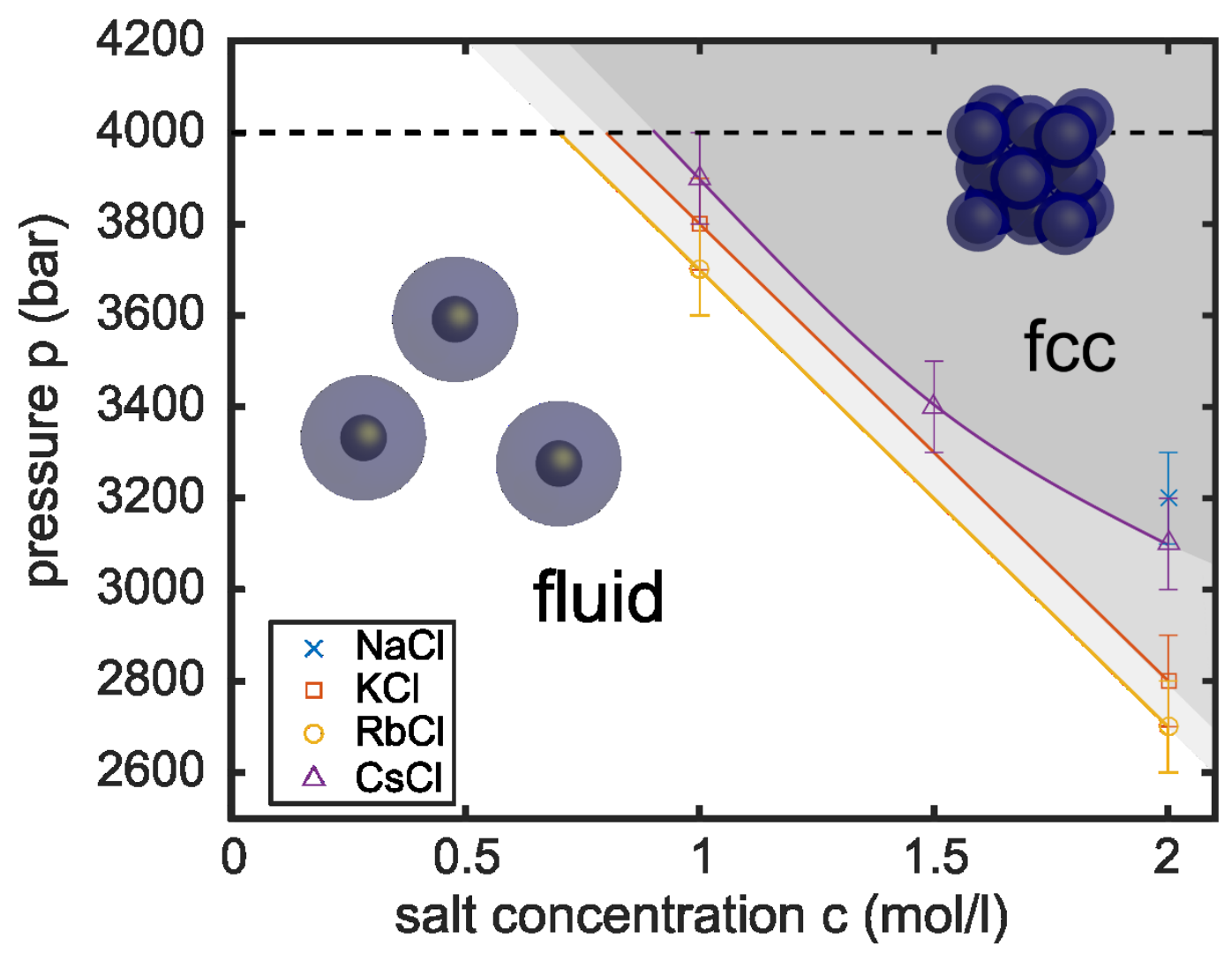

Figure 3. Pressure - salt concentration $(p-c)$ phase diagram of AuNP@PEG. For low pressures, the particles are in the liquid state, beyond a critical pressure, fcc superlattices are formed. The errorbars correspond to the pressure resolution of $\Delta p=100$ bar. The transition depends on the salt concentration as well as on the salt type. Solid lines are guide to the eye.

The transition depends on the salt type. The lowest pressure for crystallization was found for $\mathrm{RbCl}$, whereas for $\mathrm{NaCl}$ no crystal formation was observed for a salt concentration lower than 2 $\mathrm{M}$ and pressures up to 4000 bar. Interpreting the crystallization as salt-induced precipitation, the trend $\mathrm{Rb}^{+}>\mathrm{K}^{+}>\mathrm{Na}^{+}$is similar to the Hofmeister series, but $\mathrm{Cs}^{+}$deviates significantly from this. This points to specifics of the PEG-cation interaction: The salting-out-capability trend based on theoretical calculations proposed by Ren et al. follows the trend $\mathrm{K}^{+}>\mathrm{Rb}^{+}>\mathrm{Cs}^{+}>\mathrm{Na}^{+}$, which is in better agreement with our experimental findings. ${ }^{33}$ When mixing the AuNP@PEG with 1 M 
$\mathrm{CaCl}_{2}$ no crystal formation was observed within the pressure range studied, whereas for the sulfate salt $\mathrm{K}_{2} \mathrm{SO}_{4}$, crystallites with fcc structure, although less defined, were already formed at ambient conditions (Fig. S4-S5). Both observations are in accordance with expectations based on the Hofmeister series, which predicts a less pronounced salting-out capability for $\mathrm{Ca}^{2+}$ compared to alkali metal ions and a more pronounced one for sulfate compared to chloride.
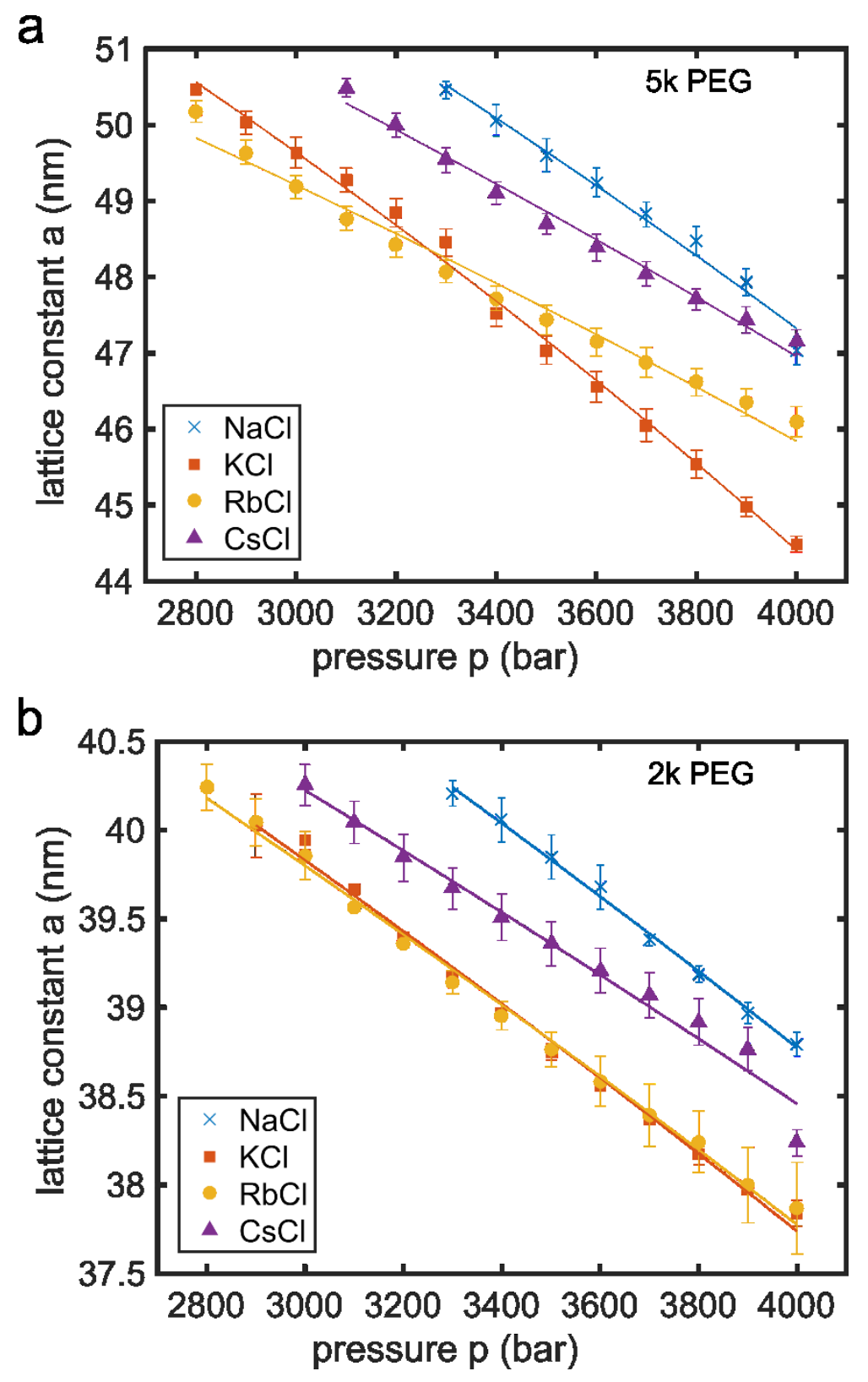
Figure 4. Effect of pressure on the formed supercrystals. Pressure-dependence of the fcc lattice constant for all salt types at the highest salt concentration studied ( $c=2 \mathrm{M})$. AuNP@PEG with a) 5k PEG, b) 2k PEG.

The lattice constant $a$ of the fcc superlattice was determined from the positions of the Bragg reflections as a function of pressure for all salt types studied at $c=2 \mathrm{M}$ (Fig. 4a), where crystals formed for all four salts. The lattice constant at the crystallization transition is found to be $a=$ $(50.5 \pm 0.3) \mathrm{nm}$ and is independent of the salt type and the crystallization pressure.

Assuming that the AuNP@PEG are closely packed in the initially formed crystallites, we can conclude from the corresponding interparticle distances $l=a(\sqrt{2})^{-1}=35.7 \mathrm{~nm}$ an effective AuNP@PEG radius of $R_{\text {eff }} \sim 18 \mathrm{~nm}$. The difference to the AuNP core radius can be attributed to an effective PEG shell thickness of $s \sim 12 \mathrm{~nm}$. This indicates, that the PEG-shells in the crystallites are still in a swollen or strongly hydrated state upon formation (for a "dry" PEG-shell of this grafting density and PEG-length $s$ would be $\sim 7 \mathrm{~nm}) .{ }^{41}$ Because of their large separation $\left(l / R_{\text {core }} \sim 6\right)$ within the crystallites, attractive van der Waals interactions of the AuNP cores can be neglected. ${ }^{1}$ The interaction potential and thus the formation of the supercrystals is dominated by the interactions of the PEG-shells only.

A further increase of pressure leads to a continuous reduction of the lattice constant, which depends on the type of cation, again indicating cation specific $\mathrm{M}^{+}$-PEG interactions. This pressure-dependence allows us to determine the effective compressibility of the lattice. The obtained values with respect to $p=4000$ bar (see table 1$)$ are larger than for water $\left(\sim 3.210^{-5}\right.$ bar $^{-}$ ${ }^{1}$ ) (extrapolated from Fine et al. ${ }^{42}$ ). This indicates that the reduction of the lattice is dominated by 
the compression of the ligand layer, whereas the gold core is not affected by pressure as evident by the absence of changes of the form factor.

Table 1. Effective compressibility of the superlattices for two PEG-ligand length and different salts types (concentration: 2M). Values in $10^{-5} \mathrm{bar}^{-1}$.

\begin{tabular}{lllll}
\hline & $\mathrm{NaCl}$ & $\mathrm{KCl}$ & $\mathrm{RbCl}$ & $\mathrm{CsCl}$ \\
\hline 2k PEG & $16.8 \pm 0.5$ & $17.4 \pm 0.4$ & $16.8 \pm 0.4$ & $14.7 \pm 0.8$ \\
$5 \mathrm{k}$ PEG & $32 \pm 2$ & $39.5 \pm 0.8$ & $23 \pm 1$ & $25 \pm 1$ \\
\hline
\end{tabular}

For a PEG-ligand length of $2 \mathrm{kDa}$, we observed similar results (Fig. S6-S7). Only the resulting lattice constant is reduced as the initial ligand layer is thinner ( $a=40 \mathrm{~nm} ; s \sim 8 \mathrm{~nm})$. The findings for the different ligand lengths strongly indicate a closed-packing of nanoparticles with hydrated PEG-shells. The effective compressibility for the $2 \mathrm{kDa}$ ligand is smaller than for the 5 $\mathrm{kDa}$ (table 1) pointing to less flexible and more densely packed ligand shell resulting in a smaller degree of hydration in accordance with previous findings. ${ }^{41}$ In addition, the effect of salt is less pronounced for the shorter chain.

Our observations of a pressure-induced phase transition from AuNP suspensions into supercrystals can be reasoned with the following scenario. At the crystallization pressure, the extended, strongly hydrated PEG-ligand shell abruptly collapses into a less swollen, but still hydrated state. We attribute this sudden structural transition to a rapid change of the solvency of the PEG-ligand in the aqueous solvent - similar to the volume phase transition well-known for 
thermogels $[43,44]$. In this scenario, the preferred interactions between the less swollen, but well-defined PEG-ligand shells of adjacent NPs are expected to be one of the driving forces to form highly ordered crystallites. A further increase of pressure continuously compresses the crystallites formed by further reducing the swollen shells. Without salt addition, no crystallization is observed in the pressure range studied. Thus, the combined effect of pressure and salt, both decreasing the solubility of PEG in water, is essential for liquid-solid phase transition. The correct choice of parameters enables the formation of NP-supercrystals in solution from a disordered, liquid state induced by hydrostatic pressure. Since PEGylation is an established method for the stabilization of nanomaterials, the demonstrated concept should be applicable for a variety of nanomaterials, including other sizes, shapes and materials. Future studies, e.g. based on pressure jumps, might reveal mechanistic insights into the supercrystal formation that will help to understand crystallization processes and to utilize pressure for the synthesis of well-defined NP supercrystals.

\section{Experimental Methods}

Experimental methods, are available in the Supporting Information.

\section{AUTHOR INFORMATION}

\section{Notes}

The authors declare no competing financial interests.

\section{ACKNOWLEDGMENT}


We acknowledge Andrew J. Smith and Tim Snow for excellent support during the SAXS measurement and Diamond Light Source for time on 22 under proposal SM16193. M.A.S thanks the Röntgen-Ångström cluster project "TT-SAS” (BMBF project number 05K16YEA). F.S. acknowledges support by the DFG via the project SCHU 3019/2-1. This work has been supported by the excellence cluster "The Hamburg Centre for Ultrafast Imaging, Structure, Dynamics, and Control of Matter at the Atomic Scale" of the DFG.

SUPPORTING INFORMATION AVAILABLE: Detailed description of sample preparation; Description of SAXS experiment and data analysis; Results for different chloride salt types and concentrations; Results for other salt types; Results for $2 \mathrm{kDa}$ ligand; Results for different particle concentration.

\section{References}

(1) Bishop, K. J. M.; Wilmer, C. E.; Soh, S.; Grzybowski, B. A. Nanoscale Forces and Their Uses in SelfAssembly. Small 2009, 5, 1600-1630.

(2) Murray, C. B.; Kagan, a. C. R.; Bawendi, M. G. Synthesis and Characterization of Monodisperse Nanocrystals and Close-Packed Nanocrystal Assemblies. Annu. Rev. Mater. Sci. 2000, 30, 545-610. (3) Boles, M. A.; Engel, M.; Talapin, D. V. Self-Assembly of Colloidal Nanocrystals: From Intricate Structures to Functional Materials. Chem. Rev. 2016, 116, 11220-11289.

(4) Macfarlane, R. J.; O’Brien, M. N.; Petrosko, S. H.; Mirkin, C. A. Nucleic Acid-Modified Nanostructures as Programmable Atom Equivalents: Forging a New "Table of Elements. Angew. Chem. Int. Ed. 2013, 52, 5688-5698.

(5) Auyeung, E.; Li, T. I. N. G.; Senesi, A. J.; Schmucker, A. L.; Pals, B. C.; La Cruz, M. O. de; Mirkin, C. A. DNA-mediated Nanoparticle Crystallization into Wulff Polyhedra. Nature 2014, 505, 73-77.

(6) Reichhelm, A.; Haubold, D.; Eychmüller, A. Ligand Versatility in Supercrystal Formation. Adv. Funct. Mater. 2017, 27, 1700361.

(7) Yang, J.; Choi, M. K.; Kim, D.-H.; Hyeon, T. Designed Assembly and Integration of Colloidal Nanocrystals for Device Applications. Adv. Mater. 2016, 28, 1176-1207.

(8) Song, R.-Q.; Cölfen, H. Mesocrystals-Ordered Nanoparticle Superstructures. Adv. Mater. 2010, 22, 1301-1330.

(9) Dreyer, A.; Feld, A.; Kornowski, A.; Yilmaz, E. D.; Noei, H.; Meyer, A.; Krekeler, T.; Jiao, C.; Stierle, A.; Abetz, V. et al. Organically Linked Iron Oxide Nanoparticle Supercrystals with Exceptional Isotropic Mechanical Properties. Nat. Mater. 2016, 15, 522-528.

(10) Pileni, M.-P. Mechanical Properties of Supracrystals. EPL 2017, 119, 37002. 
(11) Podsiadlo, P.; Krylova, G.; Lee, B.; Critchley, K.; Gosztola, D. J.; Talapin, D. V.; Ashby, P. D.;

Shevchenko, E. V. The Role of Order, Nanocrystal Size, and Capping Ligands in the Collective Mechanical Response of Three-Dimensional Nanocrystal Solids. J. Am. Chem. Soc. 2010, 132, 8953-8960.

(12) Kalsin, A. M.; Fialkowski, M.; Paszewski, M.; Smoukov, S. K.; Bishop, K. J. M.; Grzybowski, B. A. Electrostatic Self-Assembly of Binary Nanoparticle Crystals with a Diamond-Like Lattice. Science 2006, 312, 420-424.

(13) Shevchenko, E. V.; Talapin, D. V.; Kotov, N. A.; O’Brien, S.; Murray, C. B. Structural Diversity in Binary Nanoparticle Superlattices. Nature 2006, 439, 55-59.

(14) Smith, D. K.; Goodfellow, B.; Smilgies, D.-M.; Korgel, B. A. Self-Assembled Simple Hexagonal AB2 Binary Nanocrystal Superlattices: SEM, GISAXS, and Defects. J. Am. Chem. Soc. 2009, 131, 3281-3290.

(15) Ye, X.; Zhu, C.; Ercius, P.; Raja, S. N.; He, B.; Jones, M. R.; Hauwiller, M. R.; Liu, Y.; Xu, T.; Alivisatos, A. P. Structural Diversity in Binary Superlattices Self-Assembled from Polymer-Grafted Nanocrystals. Nat. Commun. 2015, 6, 10052.

(16) Maiti, S.; André, A.; Banerjee, R.; Hagenlocher, J.; Konovalov, O.; Schreiber, F.; Scheele, M. Monitoring Self-Assembly and Ligand Exchange of PbS Nanocrystal Superlattices at the Liquid/Air Interface in Real Time. J. Phys. Chem. Lett. 2018, 9, 739-744.

(17) Zaluzhnyy, I. A.; Kurta, R. P.; André, A.; Gorobtsov, O. Y.; Rose, M.; Skopintsev, P.; Besedin, I.; Zozulya, A. V.; Sprung, M.; Schreiber, F. et al. Quantifying Angular Correlations between the Atomic Lattice and the Superlattice of Nanocrystals Assembled with Directional Linking. Nano Lett. 2017, 17, 3511-3517.

(18) Geuchies, J. J.; van Overbeek, C.; Evers, W. H.; Goris, B.; Backer, A. de; Gantapara, A. P.; Rabouw, F. T.; Hilhorst, J.; Peters, J. L.; Konovalov, O. et al. In Situ Study of the Formation Mechanism of TwoDimensional Superlattices from PbSe Nanocrystals. Nat. Mater. 2016, 15, 1248-1254.

(19) Narayanan, S.; Wang, J.; Lin, X.-M. Dynamical Self-Assembly of Nanocrystal Superlattices during Colloidal Droplet Evaporation by in situ Small Angle X-Ray Scattering. Phys. Rev. Lett. 2004, 93, 135503.

(20) Li, T.; Senesi, A. J.; Lee, B. Small Angle X-ray Scattering for Nanoparticle Research. Chem. Rev. 2016, 116, 11128-11180.

(21) Abécassis, B.; Testard, F.; Spalla, O. Gold Nanoparticle Superlattice Crystallization Probed In Situ. Phys. Rev. Lett. 2008, 100, 115504.

(22) Vogel, N.; Retsch, M.; Fustin, C.-A.; Del Campo, A.; Jonas, U. Advances in Colloidal Assembly: The Design of Structure and Hierarchy in Two and Three Dimensions. Chem. Rev. 2015, 115, 6265-6311. (23) Wu, H.; Bai, F.; Sun, Z.; Haddad, R. E.; Boye, D. M.; Wang, Z.; Fan, H. Pressure-Driven Assembly of Spherical Nanoparticles and Formation of 1D-Nanostructure Arrays. Angew. Chem. Int. Ed. 2010, 49, 8431-8434.

(24) Schroer, M. A.; Westermeier, F.; Lehmkühler, F.; Conrad, H.; Schavkan, A.; Zozulya, A. V.; Fischer, B.; Roseker, W.; Sprung, M.; Gutt, C. et al. Colloidal Crystallite Suspensions Studied by High Pressure Small Angle X-ray Scattering. J. Chem. Phys. 2016, 144, 84903.

(25) Zhu, H.; Nagaoka, Y.; Hills-Kimball, K.; Tan, R.; Yu, L.; Fang, Y.; Wang, K.; Li, R.; Wang, Z.; Chen, O. Pressure-Enabled Synthesis of Hetero-Dimers and Hetero-Rods through Intraparticle Coalescence and Interparticle Fusion of Quantum-Dot-Au Satellite Nanocrystals. Journal of the American Chemical Society 2017, 139, 8408-8411.

(26) Wu, H.; Wang, Z.; Fan, H. Stress-Induced Nanoparticle Crystallization. J. Am. Chem. Soc. 2014, 136, 7634-7636. 
(27) Podsiadlo, P.; Lee, B.; Prakapenka, V. B.; Krylova, G. V.; Schaller, R. D.; Demortière, A.; Shevchenko, E. V. High-Pressure Structural Stability and Elasticity of Supercrystals Self-Assembled from Nanocrystals. Nano Lett. 2011, 11, 579-588.

(28) Schulz, F.; Vossmeyer, T.; Bastús, N. G.; Weller, H. Effect of the Spacer Structure on the Stability of Gold Nanoparticles Functionalized with Monodentate Thiolated Poly(ethylene glycol) Ligands. Langmuir 2013, 29, 9897-9908.

(29) Schulz, F.; Homolka, T.; Bastús, N. G.; Puntes, V.; Weller, H.; Vossmeyer, T. Little Adjustments Significantly Improve the Turkevich Synthesis of Gold Nanoparticles. Langmuir 2014, 30, 10779-10784.

(30) Schulz, F.; Dahl, G. T.; Besztejan, S.; Schroer, M. A.; Lehmkühler, F.; Grübel, G.; Vossmeyer, T.; Lange, $\mathrm{H}$. Ligand Layer Engineering To Control Stability and Interfacial Properties of Nanoparticles. Langmuir 2016, 32, 7897-7907.

(31) Lehmkühler, F., Schulz, F.; Schroer, M. A.; Frenzel, L.; Lange H.; Grübel G. Heterogeneous Local Order in Self-Assembled Nanoparticle Films Revealed by X-ray Cross Correlations. IUCrJ 2018, 5, 354360.

(32) Lundberg, R. D.; Bailey, F. E.; Callard, R. W. Interactions of Inorganic Salts with Poly(ethylene Oxide). J. Polym. Sci. A-1 Polym. Chem. 1966, 4, 1563-1577.

(33) Ren, C.-I.; Tian, W.-D.; Szleifer, I.; Ma, Y.-Q. Specific Salt Effects on Poly(ethylene oxide) Electrolyte Solutions. Macromolecules 2011, 44, 1719-1727.

(34) Hakem, I. F.; Lal, J.; Bockstaller, M. R. Binding of Monovalent lons to PEO in Solution: Relevant Parameters and Structural Transitions. Macromolecules 2004, 37, 8431-8440.

(35) Zámbó, D.; Radnóczi, G. Z.; Deák, A. Preparation of Compact Nanoparticle Clusters from Polyethylene Glycol-Coated Gold Nanoparticles by Fine-Tuning Colloidal Interactions. Langmuir 2015, 31, 2662-2668.

(36) Zhang, H.; Wang, W.; Akinc, M.; Mallapragada, S.; Travesset, A.; Vaknin, D. Assembling and Ordering Polymer-Grafted Nanoparticles in Three Dimensions. Nanoscale 2017, 9, 8710-8715. (37) Zhang, H.; Wang, W.; Mallapragada, S.; Travesset, A.; Vaknin, D. Macroscopic and Tunable Nanoparticle Superlattices. Nanoscale 2017, 9, 164-171.

(38) Richard L. Cook; H. E. King; Jr.; and Dennis G. Peiffer. Pressure-Induced Crossover from Good to Poor Solvent Behavior for Polyethylene Oxide in Water. Phys. Rev. Lett. 1992, 69, 3072-3075.

(39) Briscoe, B.; Luckham, P.; Zhu, S. Rheological Study of Poly(ethylene oxide) in Aqueous Salt Solutions at High Temperature and Pressure. Macromolecules 1996, 29, 6208-6211.

(40) Schroer, M. A.; Schulz, F.; Lehmkühler, F.; Möller, J.; Smith, A. J.; Lange, H.; Vossmeyer, T.; Grübel, G. Tuning the Interaction of Nanoparticles from Repulsive to Attractive by Pressure. J. Phys. Chem. $C$ 2016, 120, 19856-19861.

(41) Schulz, F.; Möller, J.; Lehmkühler, F.; Smith, A. J.; Vossmeyer, T.; Lange, H.; Grübel, G.; Schroer, M. A. Structure and Stability of PEG- and Mixed PEG-Layer-Coated Nanoparticles at High Particle Concentrations Studied In Situ by Small-Angle X-Ray Scattering. Part. Part. Syst. Charact. 2018, 35, 1700319.

(42) Fine, R. A.; Millero, F. J. Compressibility of Water as a Function of Temperature and Pressure. J. Chem. Phys. 1973, 59, 5529-5536.

(43) Das, M.; Zhang, H.; Kuacheva, E. MICROGELS: Old Materials with New Applications. Annu. Rev. Mater. Res. 2006, 36, 117-142.

(44) Freitag, R.; Garret-Flaudy, F. Salt Effects on the Thermoprecipitation of Poly-(Nisopropylacrylamide) Oligomers from Aqueous Solution. Langmuir 2002, 18, 3434-3440. 